\title{
Principles and structure of the real Multiverse: Explanation of dark matter and dark energy phenomena
}

\author{
Alexander Alexandrovich Antonov \\ Research Centre of information technology "TELAN Electronics”, Kiev, Ukraine \\ Email address: \\ telan@bk.ru
}

\section{To cite this article:}

Alexander Alexandrovich Antonov. Principles and Structure of the Real Multiverse: Explanation of Dark Matter and Dark Energy Phenomena. American Journal of Modern Physics. Vol. 4, No. 1, 2015, pp. 1-9. doi: 10.11648/j.ajmp.20150401.11

\begin{abstract}
Many hypotheses of the Multiverse have been suggested; however, they are so extraordinary that the Universes they include will never - even in the most distant future - be accessible for people. That is, they will always remain non-existent. Therefore, the hypothesis of the real Multiverse is suggested, where parallel Universes comply with the similarity principle, that is, the laws of nature governing them have much in common. This circumstance enables the inhabitants of this Multiverse to visit parallel Universes. The suggested Multiverse hypothesis relies on the adjusted special theory of relativity (the STR), where the ambiguity of the second postulate is eliminated by removing the statements on the unbreakable light speed barrier and on imaginary numbers having no physical meaning. The fact that imaginary numbers have physical meaning is supported by a theoretical and experimental investigation of oscillation processes in linear electric circuits. Since mathematics is a common and unambiguous language of science, the principle of the physical reality of imaginary numbers must be accepted as the general scientific one, true, in particular, for the STR. It is demonstrated that the Multiverse hypothesis suggested herein explains the phenomenon of the dark matter and the dark energy.
\end{abstract}

Keywords: Parallel Universes, Complex Numbers, Dark Matter, Dark Energy, Multiverse, Special Theory of Relativity, Imaginary Numbers

\section{Introduction}

The term Multiverse is understood as a hypothetical set of all Universes, including ours. Sometimes these Universes are referred to as parallel Universes, because, despite their possible infinity, they never intersect. Some of them are outlined in [1-10].

There is an assumption [7] that each Multiverse is governed by a corresponding set of consistent physical laws. The benefit of this approach is that it settles the question as to why our Universe works the way it does. On the other hand, this approach, obviously, means that other Universes are inaccessible for earthlings and, thus, they are beyond cognition.

There is also an opinion [11] that any hypotheses of other Universes (except for ours) forming the Multiverse will never be verified experimentally even in the distant future.

Nevertheless, there is a different standpoint [12-15], according to which, the Multiverse includes a set of parallel Universes abiding by the similarity principle, according to which, the physical, chemical, biological and other laws they are governed by have much in common. This leaves open the possibility for mutual visits of parallel Universes by their inhabitants. Therefore, this Multiverse is called real.

The existence of this Multiverse can be verified experimentally by organizing expeditions into these parallel worlds.

\section{Prehistory of the Real Multiverse Hypothesis}

The pre-history of the real Multiverse hypothesis starts over 500 years ago with the discovery of an imaginary unit $i=\sqrt{-1}$ by Scipione del Ferro, Niccolò Fontana Tartaglia, Gerolamo Cardano, Lodovico Ferrari and Rafael Bombelli [16]. It may have been made even earlier by Paolo Valmes, who was sentenced to death at the stake by Spanish inquisitor Tomás de Torquemada for this discovery [17].

Although at present the works of prominent mathematicians, such as Abraham de Moivre, Leonhard Euler, Jean Le Rond D'Alembert, Caspar Wessel, Pierre-Simon de Laplace, Jean-Robert Argand, Johann Carl Friedrich Gauss, Augustin 
Louis Cauchy, Karl Theodor Wilhelm Weierstrass, William Rowan Hamilton, Pierre Alphonse Laurent, Georg Friedrich Bernhard Riemann, Jan Mikusiński and many others, have developed a consistent theory of functions of a complex variable, the physical meaning of imaginary and complex numbers has not been revealed.

In 1826 Felix Savary [18] discovered the alternating electric current; later, Charles Proteus Steinmetz [19] suggested using the symbolic method to describe it. This method introduced the notion of complex frequency into the electric circuit theory; the physical meaning of this notion, however, has not been explained either in textbooks or in monographs up to now.

Finally, in the early 20th century, Joseph Larmor, Nobel Prize winner Hendrik Antoon Lorentz, Jules Henri Poincare, Nobel Prize winner Albert Einstein, as well as many other distinguished scientists, developed the special theory of relativity (STR). Its formulae describing relativistic effects at superluminal speeds had imaginary numbers, whose physical meaning physicists have been unable to explain.

However, whereas in mathematics and electric circuit theory scientists, being unable to explain the physical meaning of imaginary and complex numbers, left the question unsettled and open for further research, the STR denies their physical meaning by one of the interpretation of its second postulate [20].

Therefore the contemporary electric circuit theory at complex frequencies [21-25] does not give any descriptions of experiments, does not mention resonance at complex frequencies, and does not explain the physical meaning of complex frequencies themselves. In mathematics, algebraic equations are solved both in the set of real and in the set of complex numbers, although these solutions are sometimes mutually exclusive.

To date several hundred scientific works criticizing the STR have been published. Among the latest, [26-29] can be cited. There could have been more if influential pseudo-scientific circles had not interfered with the scientific research process. For instance, in the USSR alone, the Central Committee of the All-Union Communist Party (of Bolsheviks) and the Presidium of the Academy of Sciences adopted three decisions banning criticism of the STR - in 1934, 1942 and 1964.

However, this is natural. Karl Raimund Popper, the author of the concept of the 'open society', wrote [30] that a clash of opinions is inevitable in scientific theories and is an indispensable condition for the advancement of science. Therefore, even such a universally recognized scientific theory as the special theory of relativity cannot be regarded as a truth tout court.

In the 21 st century, physicists attempted to disprove the second postulate of the STR experimentally, for example, the MINOS experiment at the American Tevatron Collider and the OPERA experiment at the European Large Hadron Collider. These experiments argued to have registered neutrinos moving at superluminal speed. However, the physical community considered the results of the MINOS experiment not reliable enough, and the OPERA experiment was later disproved by the ICARUS experiment.

\section{Physical and Mathematical Assumption of the Real Multiverse Hypothesis}

Nevertheless, the results proving - both experimentally and theoretically - the physical reality of imaginary and complex numbers have been obtained during investigation of oscillation processes in the electric circuit theory [31-35]. These results definitely refute the above mentioned statement of the STR about imaginary numbers, as well as allows suggesting (see below) the hypothesis of the real Multiverse.

However, before moving on to the hypothesis, let us provide evidence of the physical reality of imaginary and complex numbers.

\subsection{Evidence Using Resonant Oscillation Processes}

First, let us resort to resonant oscillation processes in electric circuits, as in [31-34]. These publications demonstrate that:

- the interpretation of resonance at real frequencies is inconsistent and incomplete;

- the interpretation of resonance at complex frequencies is consistent and, thus, can rightfully be called a theory.

\subsubsection{Theoretical Evidence}

Let us make sure this is really so. For example, complex impedance of the electrical LCR-circuit in Fig. 1, according to all textbooks, is

$$
Z(j \omega)=L \frac{\omega^{1} / R C+j\left(\omega^{2}-1 / L C\right)}{\omega-j 1 / R C}=L \frac{2 \sigma_{0} \omega+j\left(\omega^{2}-\omega_{0}^{2}\right)}{\omega-j 2 \sigma_{0}}
$$

where $\omega_{0}=1 / \sqrt{L C} ; 2 \sigma_{0}=1 / R C ; j=\sqrt{-1}$ is the imaginary unit, which in the electric circuit theory is denoted in a way different from mathematics, because the symbol $\boldsymbol{i}$ is used to denote electric current.

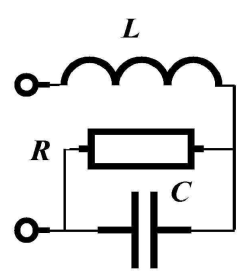

Figure 1. The LCR-circuit under investigation.

The imaginary component of this complex impedance is

$$
\operatorname{Im} Z(j \omega)=L \frac{\omega\left(4 \sigma_{0}^{2}+\omega-\omega_{0}^{2}\right)}{\omega^{2}+4 \sigma_{0}^{2}}
$$

Equating it to zero, we find, as it turns out, not one real resonance frequency, but two resonance frequencies (because the graph in Fig. 2a has two intersection points with the $\boldsymbol{\omega}$ axis) 


$$
\left[\begin{array}{l}
\omega_{r e s I}^{\prime}=0 \\
\omega_{r e s I}^{\prime \prime}=\sqrt{\omega_{0}^{2}-4 \sigma_{0}^{2}}=\omega_{0} \frac{\sqrt{Q^{2}-1}}{Q} \neq \omega_{0}
\end{array}\right.
$$

The absolute value of complex impedance (1) is

$$
|Z(j \omega)|=L \sqrt{\frac{4 \sigma_{0}^{2} \omega^{2}+\left(\omega^{2}-\omega_{0}^{2}\right)^{2}}{\omega^{2}+4 \sigma_{0}^{2}}}
$$

Testing for extremum the radicand, i.e., solving the equation

$$
\begin{aligned}
& \frac{d}{d \omega}\left[\frac{4 \sigma_{0}^{2} \omega^{2}+\left(\omega^{2}-\omega_{0}^{2}\right)^{2}}{\omega^{2}+4 \sigma_{0}^{2}}\right]= \\
& =\frac{2 \omega\left[\omega^{4}+8 \sigma_{0}^{2} \omega^{2}+\left(16 \sigma_{0}^{4}-8 \sigma_{0}^{2} \omega_{0}^{2}-\omega_{0}^{4}\right)\right.}{\left(\omega^{2}+4 \sigma_{0}^{2}\right)^{2}}=0
\end{aligned}
$$

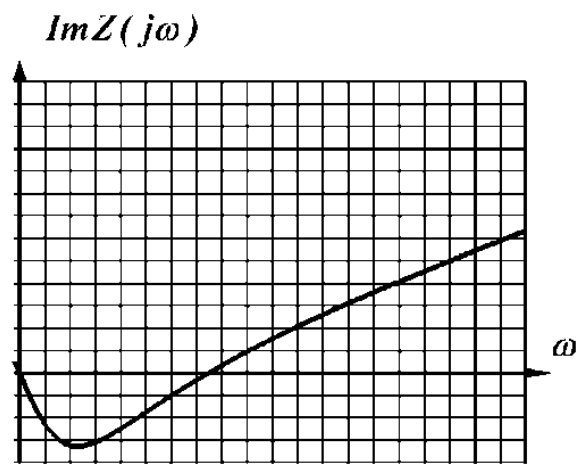

a

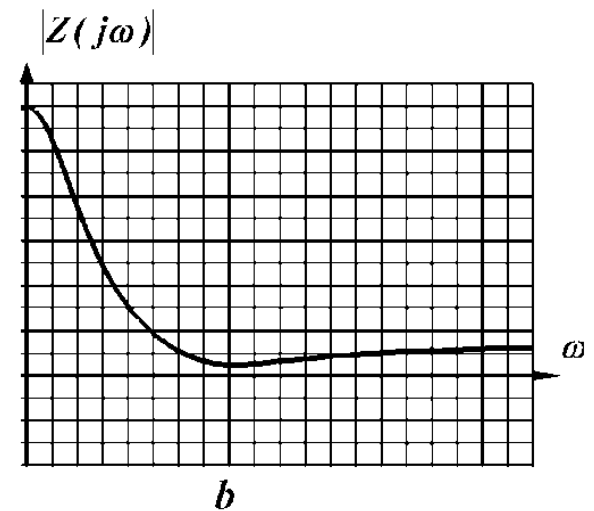

Figure 2. Graphs of functions (2) and (4).

$$
\frac{d^{2} I}{d t^{2}}+\frac{1}{R C} \frac{d I}{d t}+\frac{1}{L C} I=\frac{1}{L} \frac{d U}{d t}+\frac{1}{L C R} U
$$

where $\boldsymbol{U}$ is the input electric current applied to the circuit; $\boldsymbol{I}$ is the electric current in the LCR-circuit; then the corresponding characteristic algebraic equation has the form

$$
p^{2}+\frac{1}{R C} p+\frac{1}{L C}=p^{2}+2 \sigma_{0} p+\omega_{0}^{2}=0
$$

and its solutions are $p_{1.2}=-\sigma_{0} \pm j \sqrt{\omega_{0}^{2}-\sigma_{0}^{2}}$. Consequently, the real frequency of free oscillations turns to be

$$
\omega_{\text {free }}=\operatorname{Im} p_{1,2}=\sqrt{\omega_{0}^{2}-\sigma_{0}^{2}}=\omega_{0} \frac{\sqrt{4 Q^{2}-1}}{2 Q} \neq \omega_{0}
$$

Thus, this LCR-circuit has, in fact, several real resonance frequencies corresponding to the different attributes of the resonance. Moreover, none of these real resonance frequencies equals the real frequency of free oscillations, although free oscillations would seem to exist at more energetically efficient, i.e., at the resonance frequency (but which one of them?). And all this isn't explained anywhere. two more real resonance frequencies are found (since the graph in Fig. 2b has two extremums)

$$
\left[\begin{array}{l}
\omega_{\text {res } 2}^{\prime}=0 \\
\omega_{\text {res } 2}^{\prime \prime}=\sqrt{\omega_{0} \sqrt{\omega_{0}^{2}+8 \sigma_{0}^{2}-4 \sigma_{0}^{2}}}= \\
=\omega_{0} \frac{\sqrt{Q \sqrt{Q^{2}+2}-1}}{Q} \neq \omega_{0}
\end{array}\right.
$$

Let us also find the real frequency of free oscillations for this LCR-circuit. Since processes in it are described with a differential equation

Similar results are obtained using precise mathematical analysis of other LCR-circuits, as well.

\begin{tabular}{|c|c|c|c|}
\hline$Q$ & $\omega_{\text {free }} / \omega_{0}$ & $\omega_{r e s 1}^{\prime \prime} / \omega_{0}$ & $\omega_{r e s 2}^{\prime \prime} / \omega_{9}$ \\
\hline 2 & 0,9682458 & 1,1547005 & 1,0128719 \\
\hline 3 & 0,9860133 & 1,0606602 & 1,0027964 \\
\hline 4 & 0,9921567 & 1,0327956 & 1,0009212 \\
\hline 5 & 0,9949874 & 1,0206207 & 1,000385 \\
\hline 6 & 0,9965217 & 1,0141851 & 1,0001878 \\
\hline 7 & 0,9974457 & 1,0103630 & 1,0001021 \\
\hline 8 & 0,9980450 & 1,0079053 & 1,0000601 \\
\hline 9 & 0,9984556 & 1,0062306 & 1,0000376 \\
\hline 10 & 0,9987492 & 1,0050378 & 1,0000248 \\
\hline 20 & 0,9996875 & 1,0012523 & 1,0000016 \\
\hline 30 & 0,9998611 & 1,0005560 & 1,0000003 \\
\hline 40 & 0,9999219 & 1,0003126 & 1,0000001 \\
\hline
\end{tabular}

However, it is easy to see (Table 1) that the differences between resonance frequencies $\omega_{\text {res } 1}^{\prime \prime}$ and $\omega_{\text {res } 2}^{\prime \prime}$, as well as the frequency of free oscillations $\boldsymbol{\omega}_{\text {free }}$ and the frequency $\omega_{0}$ are quite insignificant. At the same time, the existence of these differences, as well as the existence of resonance frequencies $\omega_{r e s 1}^{\prime}$ and $\omega_{r e s 2}^{\prime}$ require an explanation.

Table 1. Resonance frequencies and frequency of free oscillations. 


\begin{tabular}{|c|c|c|c|}
\hline$Q$ & $\omega_{\text {free }} / \omega_{0}$ & $\omega_{r e s 1}^{\prime \prime} / \omega_{0}$ & $\omega_{r e s 2}^{\prime \prime} / \omega_{9}$ \\
\hline 50 & 0,9999500 & 1,0002001 & 1,0000000 \\
\hline 60 & 0,9999653 & 1,0001389 & 1,0000000 \\
\hline 70 & 0,9999745 & 1,0001021 & 1,0000000 \\
\hline 80 & 0,9999805 & 1,0000781 & 1,0000000 \\
\hline 90 & 0,9999846 & 1,0000617 & 1,0000000 \\
\hline 100 & 0,9999875 & 1,0000500 & 1,0000000 \\
\hline
\end{tabular}

By the way, in the OPERA experiment, the difference between the velocity of a neutrino and the speed of light was also quite insignificant and comparable to an experimental error. Nevertheless, in less than half a year, several dozens of scientific works were devoted to explaining and refuting the results of the OPERA experiment. And both situations, in fact, deal with the same problem: the answer to the question whether imaginary numbers are physically real.

It turns out that they are, because resonance is physically real at complex frequencies $p_{\text {res }}=p_{\text {free }}=-\sigma_{0} \pm$ $\pm j \sqrt{\omega_{0}^{2}-\sigma_{0}^{2}}$, which correspond to the solutions of the characteristic algebraic equation (8). Refs. [32-34] provide a detailed validation of this statement using Cassini ovals and vector diagrams at complex frequencies. In particular, all the aforementioned unclear inconsistencies of interpretation of resonance at real frequencies are explained. Let us not waste the reader's time by repeating them here.

\subsubsection{Experimental Evidence}

Now let us move on to the scientists' Ultima ratio - the practical physical experiments (that have nothing to do with the so-called mental experiments - the latter is mere reasoning and may very well turn out to be wrong), or, to be more precise, to one of the experiments described in [32-34]. Fig. 3 shows three electric circuits with similar processes. Let us make sure this is really so.
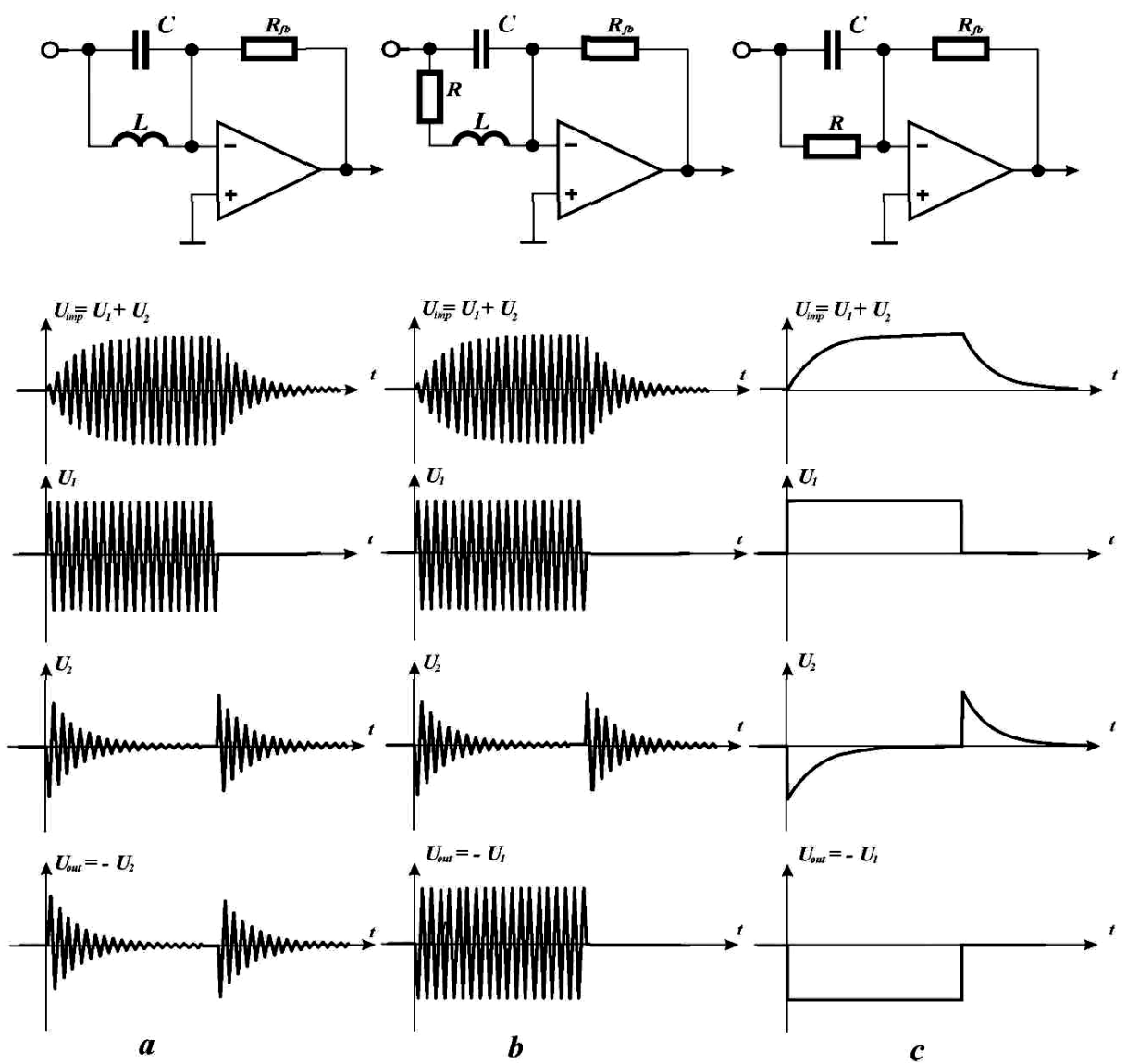

Figure 3. Rejection filters of complex frequencies.

In the electric circuit shown in Fig. 3a, the oscillating LC-circuit is connected to the inverting input of an operational amplifier. The transfer function of this circuit is

$$
k(p)=-R_{f b} C \frac{p^{2}+\omega_{0}^{2}}{p}
$$

where $p$ is complex frequency; $\boldsymbol{\omega}_{0}=1 / \sqrt{\boldsymbol{L} C}$ is the real value of a capacitor which is part of the oscillatory circuit; resonance frequency of the electric LC-circuit; $\boldsymbol{C}$ is the $\boldsymbol{L}$ is the inductance of the coil which is part of the oscillatory circuit; $\boldsymbol{R}_{f b}$ is the resistance of the feedback resistor connected between the output and the inverting input of the operational amplifier.

Therefore, if radio pulses $\boldsymbol{U}_{\text {inp }}(\boldsymbol{t})$ are fed to the input of this circuit at a complex resonance frequency of the LC-circuit, at the output the signal in the form of exponential 
radio pulses $U_{\text {out }}(t)$ is obtained. This is due to the fact that the input signal $U_{\text {inp }}(t)$ is actually a sum of two signals rectangular radio pulses with steep edges $\boldsymbol{U}_{I}(t)$ at complex-conjugate frequencies $p= \pm j \omega_{0}$ and exponential radio pulses $\boldsymbol{U}_{2}(\boldsymbol{t})$ at complex-conjugate frequencies $p=-\sigma \pm j \omega_{0}$. However, in the LC-circuit, resonance under the influence of rectangular radio pulses is observed at the input of the operational amplifier, because of what the magnitude of the transfer function (10) of the circuit under investigation at complex-conjugate frequencies $p= \pm j \omega_{0}$ is zero. This is why rectangular radio pulses $\boldsymbol{U}_{I}(\boldsymbol{t})$ do not reach the output. At the same time, the magnitude of the transfer function at complex-conjugate frequencies $p=-\sigma_{0} \pm j \omega_{0}$ is non-zero. This accounts for the fact that only inverted exponential radio pulses reach the output, i.e. $U_{\text {out }}(t)=-U_{2}(t)$.

In the electric circuit in Fig. $3 b$, on the contrary, rectangular radio pulses $U_{I}(t)$ are found at the output, whereas exponential radio pulses $U_{2}(t)$ are not, because the circuit's transfer function has the form

$$
k(p)=-R_{f b} C \frac{p^{2}+2 \sigma_{o} p+\omega_{0}^{2}}{p}
$$

This is why at complex-conjugate frequencies $p=-\sigma_{0} \pm j \omega_{0}$ this transfer function is zero, and at complex-conjugate frequencies, $\boldsymbol{p}= \pm j \omega_{0}$ it is non-zero, which accounts for the results obtained. Consequently, in the LCR-circuit, resonance under the influence of exponential radio pulses is observed at the input of the operational amplifier.

As can be seen, in both electric circuits the necessary result is provided for by tuning the input oscillatory circuit connected to the inverting input of an operational amplifier to resonance with the frequency of the signal that is not supposed to reach the output. Consequently, both electric circuits are band-rejection filters of complex frequencies. Moreover, these rejection filters provide for the result impossible if any known filters of real frequencies are used, because the spectra of the rectangular radio pulses $U_{I}(t)$ and the exponential radio pulses $\boldsymbol{U}_{2}(\boldsymbol{t})$ overlap significantly. At that, since the spectrum of the rectangular radio pulse $U_{I}(t)$ is broader than that of the exponential radio pulse $U_{2}(t)$, the result shown in the scheme in Fig. 3a seems even more confusing

It is interesting to note that similar results can be obtained if radio pulses are replaced by video pulses, i.e., resonance can be observed under the influence of exponential video pulses at the complex frequency $p=-\sigma_{0}$. Indeed, the transfer function of the electric circuit in Fig. 3c, where an inverting input of an operational amplifier is connected to a parallel electric RC-circuit, is

$$
k(p)=-R_{f b} C\left(p+\sigma_{0}\right)
$$

where $\sigma_{0}=1 / R C$.

Therefore, if video pulses $U_{\text {inp }}(t)$ with low-angle edges are fed to the input, video pulses $\boldsymbol{U}_{\text {out }}(\boldsymbol{t})$ with steep edges are recorded at the output. This is accounted for by the fact that this input signal is, in fact, a sum of two signals $U_{\text {inp }}(t)=U_{1}(t)+U_{2}(t)$, i.e., the rectangular video pulses with steep edges $\boldsymbol{U}_{I}(\boldsymbol{t})$ at the complex frequency $p=0$ and the exponential video pulses $\boldsymbol{U}_{2}(\boldsymbol{t})$ at the complex frequency $p=-\sigma_{0}$. However, the magnitude of the transfer function of the circuit under investigation at the complex frequency $p=-\sigma_{0}$ is zero. This is why the exponential video pulses $\boldsymbol{U}_{2}(\boldsymbol{t})$ do not reach the output. At the same time, the magnitude of the transfer function of the same circuit at the complex frequency $\boldsymbol{p}=\boldsymbol{0}$ is non-zero. Therefore, only inverted rectangular video pulses with steep edges $-\boldsymbol{U}_{\boldsymbol{I}}(\boldsymbol{t})$ reach the output. This result cannot be accounted for by the electric circuit theory at real frequencies, either.

Thus, the above allows concluding that in nature resonance exists not at real, but at complex frequencies, and that complex frequency is physically real.

\subsection{Evidence Using Transient Oscillation Processes}

The physical reality of complex frequencies can be proven by investigating transient oscillation processes, as well [12, 13, 35]. Moreover, in the latter case, the argumentation is very simple. Indeed, all engineers know that in order to determine the particular type of transient processes, the differential equations describing them

$$
a_{n} \frac{d^{n} y}{d t^{n}}+a_{n-1} \frac{d^{n-1} y}{d t^{n-1}}+\ldots+a_{0} y=-b_{m} \frac{d^{m} x}{d t^{m}}+b_{m-1} \frac{d^{m-1} x}{d t^{m-1}}+\ldots+b_{0} x
$$

where $\boldsymbol{x}(\boldsymbol{t})$ is the input action (or the input signal);

$\boldsymbol{y}(\boldsymbol{t})$ is the response to the action (or the output signal);

$\boldsymbol{a}_{n}, \boldsymbol{a}_{n-1}, \ldots, \boldsymbol{a}_{0}, \boldsymbol{b}_{m}, \boldsymbol{b}_{m-1}, \ldots, \boldsymbol{b}_{0}$ are constant coefficients;

$n, n-1, \ldots, 0, m, m-1, \ldots, 0$ is the order of derivatives;

are replaced (unless the operational method of solving differential equations is used) for the corresponding characteristic algebraic equations

$$
a_{n} p^{n}+a_{n-1} p^{n-1}+\ldots+a_{0}=0
$$

where $\boldsymbol{a}_{n}, \boldsymbol{a}_{n-1}, \ldots, \boldsymbol{a}_{0}$ are the same constant coefficients as in equation (13); $\boldsymbol{n}, \boldsymbol{n}-\mathbf{1}, \boldsymbol{n}-\mathbf{2}, \ldots, \mathbf{1 , 0}$ are the exponents with a magnitude equal to the order of the corresponding derivatives in the differential equation (13); $\boldsymbol{p}$ is a variable, which, in case it takes values in the form of complex numbers $-\sigma \pm i \omega$, is often referred to as complex frequency; and solved.

The solutions are always found only in the set of complex 
numbers, because various transient processes always exist; however, the solutions of algebraic equation (14) corresponding to these transient processes can always be found only in the set of complex numbers, and may take the form of complex numbers which correspond to an oscillation transient process.

If the characteristic algebraic equations were solved in the set of real numbers, as is sometimes the case in algebra, transient oscillation processes would not be supposed to exist in nature, because they would have no corresponding solution of the characteristic algebraic equation (14) in the form of real numbers. So, there would be no tsunami, no church bells would toll and so on.

Consequently, oscillation transient processes, including those observed in nature, prove the physical reality of complex frequencies.

The physical reality of complex frequencies results in the physical reality of its derivative quantities: the impedance and conductance of electric circuits, voltage drops and electric currents flowing through electric circuits, and the power and stored energy of electric circuits. Since oscillation processes can be not only electricity, but acoustic, hydraulic, mechanical, and of other kinds as well, complex quantities of other physical kinds will be physically real, as well.

\section{Structure of the Real Multiverse: Dark Matter and Dark Energy}

Thus, despite the fact that the MINOS and OPERA experiments failed to prove the physical reality of imaginary numbers, it was proven by the investigation of oscillation processes in linear LCR-circuits. Since nature is a single entity, science must also be integral and consistent. Therefore, the principle of the physical reality of imaginary numbers must be recognized as a general scientific principle, and, thus, it must hold true not only for electric circuit theory [31-35], but for the STR [12-15, 36], quantum mechanics [37, 38], and other theories as well.

Therefore, the theories that were developed based on the erroneous assumption of the STR on imaginary numbers having no physical meaning must be adjusted accordingly.

Let us demonstrate how this can be done, for example, in the STR.

To this end, obviously, it is necessary to explain the meaning of relativistic formulae at superluminal speeds; at that, it is enough to discuss one of them, for example, the Lorentz-Einstein formula

$$
m=\frac{m_{0}}{\sqrt{1-(v / c)^{2}}}
$$

where $\boldsymbol{m}_{\boldsymbol{0}}$ is rest mass;

$\boldsymbol{m}$ is the relativistic mass of a moving body (e.g., an elementary particle);

$v$ is the velocity of the body;

$c$ is the light speed.
It follows from (15) that at subluminal speeds the masses of elementary particles referred to as tardyons (or bradyons) are measured with real numbers, and at hyperluminal speeds the masses of elementary particles called tachyons are measured with imaginary numbers. It is noteworthy that tachyons cannot be registered from our tardyon (let us refer to it like that) Universe, because they are found elsewhere (in other words, the statement on the existence of tachyons is a hint to the existence of the Multiverse; another hint is the discovery of the dark matter and the dark energy - see below); and this other place can be referred to as the tachyon Universe.

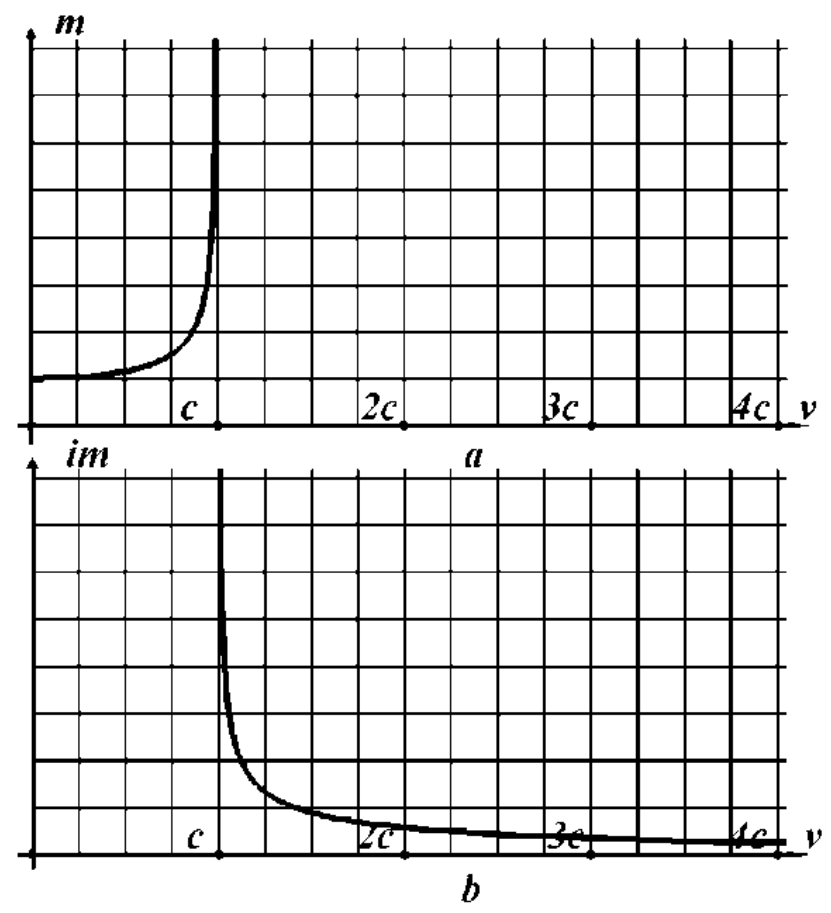

Figure 4. Graphs of functions (15).

It can be assumed that the so-called aliens, i.e., inhabitants of other Universes, have been somehow visiting the Earth from an adjacent tachyon Universe (or tachyon Antiverse see below). However, for these alien visits take place, adjacent Universes must comply with the 'similarity principle', i.e., their governing physical, chemical, biological and other regularities must be much the same as ours, although some differences are possible. For instance, in other Universes, time can flow in different directions than in ours: in the opposite direction, perpendicularly, or any other direction.

However, as can be seen (Fig. 4), the Lorentz-Einstein formula does not comply with the 'similarity principle'. Therefore, it must be adjusted as follows

$$
m=\frac{(i)^{q} m_{0}}{\sqrt{1-(v / c-q)^{2}}}=\frac{(i)^{q} m_{0}}{\sqrt{1-(w / c)^{2}}}
$$

where $q=\lfloor v / c\rfloor$ is the discrete 'floor' function of the 
argument $\boldsymbol{v} / \boldsymbol{c} ; \boldsymbol{w}=\boldsymbol{v}-\boldsymbol{q c}$ is the local, for each Universe, velocity (Fig. 5e,f,g,h), which can take values in the range $\boldsymbol{0} \leq \boldsymbol{w}<\boldsymbol{c} ; \boldsymbol{v}$ is the velocity measured from our tardyon Universe, which can thus be referred to as the tardyon velocity (Fig. 5a,b,c,d).

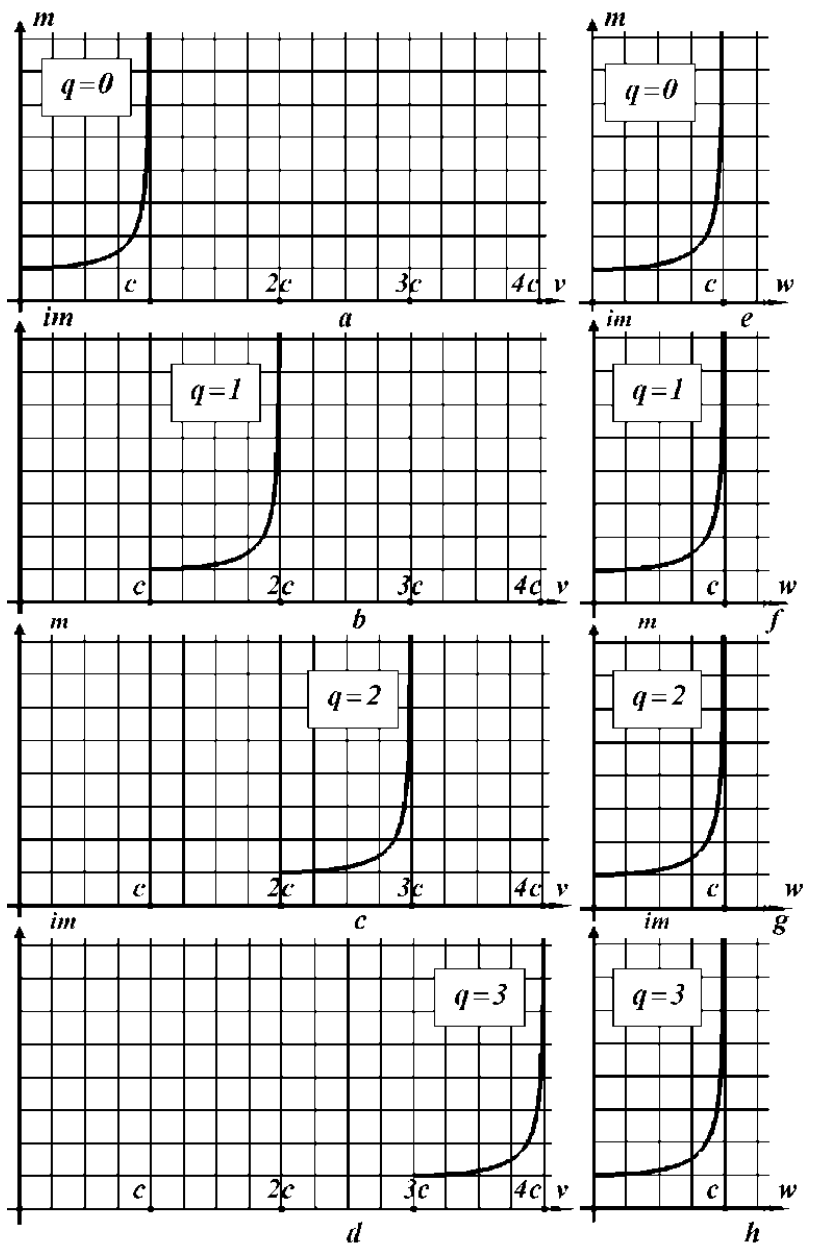

Figure 5. Graphs of functions (16).

Other relativistic formulae of the STR, naturally, have the same shortcoming and can be adjusted in a similar way.

However, let us move back to formula (16). As can be seen, at $\boldsymbol{q}=\mathbf{0}$ it corresponds to the tardyon Universe, and at $\boldsymbol{q}=\boldsymbol{1}$ to the tachyon Universe. Fig. 5 suggests that there must be at least two more parallel Universes: corresponding to $q=2$ and $q=3$. It is logical to refer to them as the tardyon Antiverse and the tachyon Antiverse, because they correspond to the values of mass, time, and other quantities defined by the relativistic formulae, measured with negative real numbers and negative imaginary numbers.

Moreover, since above, the principle of the physical reality of complex (not only imaginary) numbers has been proven, there may be numerous other parallel Universes that correspond to intermediate, i.e., non-integer, values of $\boldsymbol{q}$ tardyon-tachyon, tachyon-antitardyon, and so on.

Thus, in the Multiverse structure proposed here, the tardyon Universe and the tardyon Antiverse are never adjacent, but are divided by the tachyon Universe and the tachyon Antiverse (Fig. 5). Similarly, the tachyon Universe and the tachyon Antiverse are never adjacent, because they are separated by the tardyon Universe and the tardyon Antiverse (Fig. 5). Thus, any annihilation of the corresponding Universes and Antiverses is prevented.

Then, to $q=4$ formula (16) once again corresponds the tardyon Universe. However, this can be either our Universe or another tardyon Universe. In the former case, the Multiverse has a ringed structure, and in the latter case, a helical structure. Depending on the range of variation $\boldsymbol{q}$, the number of coils of a helix can be either finite (then the Multiverse has the structure of a screw collar) or infinite (then the Multiverse has the structure of an infinite-turn screw). Further research has yet to reveal the actual structure of the Multiverse and the corresponding range of variation of $\boldsymbol{q}$.

In the context of the discussion, it is appropriate to mention that, according to the astrophysical observations, the measured mass of all visible objects of the Universe is approximately $4,4 \%$ of its total mass. In view of the above, it must be clear that, in fact, the total mass of our tardyon Universe is meant, which is referred to as the visible Universe, and the total mass of the whole Multiverse and not the Universe. In the Multiverse, parallel Universes are, naturally, invisible to the inhabitants of our tardyon Universe, in particular, for earthlings. According to the same observations, the other, invisible part of the Universe includes 'the dark matter' (23\%) and 'the dark energy' $(72,6 \%)$. However, taking into account the discussion above, it can be assumed that they're registered physical manifestations, in fact, are caused by the existence of many other, in addition to ours, parallel Universes in the Multiverse. If this is true, then the number of parallel Universes, it includes must be $100 \% / 4,4 \% \approx 22,7$. In the helical Multiverse structure the number of parallel Universes must be integral and multiple of four; besides, masses of different parallel Universes are not supposed to be exactly identical; therefore, the numbers closest to the one found above are $q=\mathbf{2 0}$ or $q=\mathbf{2 4}$. Thus, a conclusion can be made that the number of coils of a helix is either 5 or 6 . Therefore, the phenomenon of the dark matter and the dark energy is a mere consequence of the existence of other Universes in the Multiverse, and, thus, proves the existence of the latter.

At the same time, the above-mentioned parallel Universes are not steady with respect to each other in the Multiverse, but sort of float in additional spatial dimensions. Therefore, sometimes they touch each other, and in some places even partially penetrate each other. Then, at the places of these mutual penetrations, certain transition areas, or portals (they have nothing to do with the so-called 'wormholes'), appear.

Now it is possible to explain how elementary particles, living beings, and other physical objects can make transitions from one parallel Universe to an adjacent parallel Universes, where tardyon velocities, according to (16), differ from the value of the speed of light $c$. It turns out that in nature, instead of breaking the barrier of the speed of light in accordance with the relativistic formulae of the STR, portals are used, and there are quite a few of them in the Multiverse.

This situation is similar to the way one does not have to go through the wall to move from one room of our apartment 
into another: one uses the doors that were made for the purpose.

In portals, during a transition from one parallel Universe into an adjacent parallel Universe, the value $\boldsymbol{q}$ gradually changes from one integral value to another. This is similar to the gradual transition from air into water and backwards during sea bathing.

It must be kept in mind that visiting other parallel Universes through labyrinths of portals is dangerous, because relativistic time with them, according to the formula similar to (16), for an outside observer (for instance, from our Universe) can flow in different directions (nevertheless, each local time in each Universe flows from the past to the future) with respect to our tardyon time. Other, yet unknown, dangers are also possible.

\section{Conclusion}

The research presented herein leaves many questions unanswered. This is quite natural, because source experimental information on the research of portals and parallel Universes is lacking. It can be obtained only after visiting and investigating, first, the portals, and then adjacent parallel Universes.

Investigation and exploration of the resources of the Multiverse would definitely change human civilization drastically. New knowledge and new material resources would inevitably facilitate the intellectual and economic advancement of humanity.

At last, in the Multiverse the human civilization will be able to escape in case of threat to its existence.

\section{References}

[1] Lewis, D. On the Plurality of Worlds. Basil Blackwell, Oxford (1986)

[2] Green, B. The Elegant Universe: Superstrings, Hidden Dimensions and the Quest for the Ultimate Theory. W. W. Norton \& Company, NY (2004)

[3] Deutsch, D. The structure of the multiverse. Proceedings of the Royal Society A, 458, 2911-2923 (2002).

[4] Tegmark, M. Parallel universes. Scientific American. 288(5), 40-51 (2003)

[5] Ellis, G. F. R., Kirchner, U., Stoeger, W. R. Multiverses and physical cosmology. Monthly Notices of the Royal Astronomical Society. 347(3), 921-936 (2004).

[6] Steinhardt, P. J., Turok, N. Endless Universe: Beyond the Big Bang. Doubleday, NY (2007)

[7] Tegmark, M. The mathematical universe. Foundation of Physics. 38(2), 101-150 (2008)

[8] Carr, B. (ed.). Universe or Multiverse? Cambridge University Press (2009)

[9] Lucash, V. N., Mikheyeva, E. V. Physical Cosmology. Physmathlit, Moscow (2010)
[10] Greene, B. The Hidden Reality: Parallel Universes and the Deep Laws of the Cosmos. Knopf, NY (2011)

[11] Ellis, G. F. R. Does the multiverse really exist? Scientific American. 305, 38-43 (2011)

[12] Antonov, A.A. Structure of the Multiverse. British Journal of Science. 2(2), 51-60 (2011)

[13] Antonov, A.A. Discovery of the Real Multiverse. Encyclopedia of Russian Thought: Reports to Russian Physical Society. 16(3), 3-20 (2012)

[14] Antonov, A. A. Earth. Portals. Parallel universes. American Journal of Scientific and Industrial Research. 3(6), 464-473 (2012)

[15] Antonov, A. A. Cognition of the multiverse as a factor facilitating the development of humanity. Russian Physical Thought Journal. 1(12) 6-77 (2013)

[16] Weisstein E.W. (2005). The CRC Concise Encyclopedia of Mathematics. 3-rd ed. CRS Press. Roca Raton, FL. (2005).

[17] Beckmann, P. A History of $\pi$. 5th ed. St. Martin's Press, NY (1976).

[18] Blanchard, J. The history of electrical resonance. Bell System Technical Journal, 20(4) 415-433 (1941)

[19] Steinmetz, C. P., Berg E. J. Theory and Calculation of Alternating Current Phenomena. Electrical World and Engineer Inc., NY (1900)

[20] Antonov, A.A. Verification of the second postulate of the special relativity theory. Global Journal of Science Frontier Research A: Physics and Space Science. 14(3). 51-59 (2014)

[21] Bode, H.W. Network Analysis and Feedback Amplifier Design. Van Nostrand, Princeton (1945)

[22] Franco, S. Electric Circuits Fundamentals. Oxford University Press. (1994)

[23] Balabanian, N. Electric Circuits. McGraw Hill, NY (1994)

[24] Cogdell, J.R. Foundations of Electric Circuits. Prentice Hall, NJ (1999)

[25] Bell, D.A: Fundamentals of Electric Circuits. Oxford University Press (2009)

[26] Artekha, S.N. Criticism of the Basics of the Relativity Theory. 2nd ed. LKI Publishing, Moscow (2007)

[27] Goldsmith, D. Einstein's Greatest Blunder?: The Cosmological Constant and Other Fudge Factors in the Physics of the Universe. Harvard University Press, Cambridge, Mass. (1997)

[28] Kalinin, L.A. Einstein's Cardinal Errors. Editorial URSS Publishing, Moscow (2003)

[29] Albert, D.Z., Galchen, R. Was Einstein wrong? A quantum threat to special relativity. Scientific American, 300(3), 32-39 (2009)

[30] Popper, K.R. Conjectures and Refutations. The Growth of Scientific Knowledge. Routledge, London (2002)

[31] Antonov, A.A. Buzhev, V.M.: Means of rising deflecting currents for spiral beam sweep on the CRT screen. Patent of USSR \# 433650 (1974) 
[32] Antonov, A.A. Physical reality of resonance on complex frequencies. European Journal of Scientific Research. 21(4), 627-641 (2008)

[33] Antonov, A.A. Resonance on real and complex frequencies. European Journal of Scientific Research. 28(2), 193-204 (2009)

[34] Antonov A.A.: Oscillation processes as a tool of physics cognition. American Journal of Scientific and Industrial Research. 1(2). 342-349 (2010)

[35] Antonov, A.A. Solution of algebraic quadratic equations taking into account transitional processes in oscillation systems.
General Mathematics Notes. 1(2), 11-16 (2010)

[36] Antonov, A.A. Correction of the special theory of relativity: Physical reality and nature of imaginary and complex numbers. American Journal of Scientific and Industrial Research. 5(2), 40-52 (2014)

[37] Spenkov, G.P. Physical meaning of imaginary unit i. Encyclopedia of Russian Thought: Reports to Russian Physical Society. 20(2), $70-81$ (2013)

[38] Ahkosov Ju.L. Florensky physical plane as the basis for the macrocosm substratum. Encyclopedia of Russian Thought: Reports to Russian Physical Society. 23(2). 46 - 82. 